\title{
Competencias genéricas, competencias para la vida
}

\author{
Generic Skills, skills for Life
}

Diana Marcela Sánchez Echeverri

Universidad Metropolitana de Ciencia y Tecnología. Ciudad de Panamá, Panamá

Recibido: 05/08/2021 Revisado: 20/09/2021_Aceptado: 08/10/2021 Publicado: 31/10/2021

\section{RESUMEN}

Las nuevas políticas mundiales donde la educación se promueve como eje central en el progreso social, económico, tecnológico y cultural de un país o región, han generado que los sistemas educativos cambien su perspectiva y sean planteados alrededor de estrategias donde prime la calidad de los aprendizajes por encima de conocimientos teóricos. La globalización de mercados, las tecnologías de la información, la multiculturalidad, y la innovación en los diferentes ámbitos han influenciado estos cambios mundiales, los cuales buscan que los resultados en los aprendizajes se produzcan de forma eficiente y veraz. Desde este punto de vista, se hace necesaria una educación transformadora de vidas, capaz de desarrollar habilidades para un mundo continuamente cambiante, y en permanente competitividad, una educación basada en valores humanos y medio ambientales, enmarcada por la equidad, la inclusión, la calidad, pertinencia y la permanencia en el sistema, no por la obligatoriedad sino por la necesidad de ser partícipe de este nuevo mundo, enfocada en la necesidad de aprender a aprender, en el saber hacer ante las diferentes situaciones presentadas, en el autoaprendizaje que garantice que el sujeto permanezca en formación continua a lo largo de su vida y en el saber ser en la interacción por las diversas relaciones laborales, personales y sociales con que se deberá encontrar a lo largo de su vida. Así, surge la educación basada en competencias, una educación que garantice transformación en la vida gracias a los aprendizajes valiosos que adquiera el sujeto durante toda su formación. La educación basada en competencias busca formar personas que, si bien estén altamente calificadas en su saber hacer, sean personas con comunicación asertiva, capaces de trabajar en equipo, innovadores, emprendedores, con alta capacidad en la resolución de problemas, y con fuertes valores éticos. Los últimos acontecimientos mundiales, han dejado a la vista las debilidades que aun hoy se tienen frente a la falta de competencias, pero han mostrado como las mismas generan los cambios necesarios para evolucionar como sociedad ante los desafíos que enfrenta el ser humano.

Palabras clave: competencias, genéricas, aprendizaje. 


\section{ABSTRACT}

The new world policies where education is promoted as a central axis in the social, economic, technological and cultural progress of a country or region, have generated educational systems to change their perspective and to be raised around strategies where the quality of learning prevails above theoretical knowledge. The globalization of markets, information technologies, multiculturalism, and innovation in different areas have influenced these world changes, which seek for the results in learning to occur efficiently and truthfully. From this point of view, a life-transforming education is necessary, capable of developing skills for a continuously changing world, and in permanent competitiveness, an education based on human and environmental values, framed by equity, inclusion, quality, relevance and permanence in the system, not because of the obligation but because of the need to be a participant in this new world, focused on the need to learn how to learn, on the know-how in the different situations presented, on self-learning that guarantees that the individual remains in continuous training throughout their life and in knowing how to be in the interaction through the various work, personal and social relationships that he must encounter throughout their life. Thus, competency-based education arises, an education that guarantees transformation in life thanks to the valuable learning acquired by the individual throughout their training. Competency-based education seeks to train people who, although highly qualified in their know-how, are also people with assertive communication, capable of teamwork, innovative, enterprising, with high capacity in solving problems, and with strong ethical values. The latest world events have exposed the weaknesses that still exist today because of the lack of skills, but have shown how they generate the necessary changes to evolve as a society in the face of the challenges that human beings face.

Keywords: competences, generic, learning.

\section{INTRODUCCIÓN}

A partir del último tercio del siglo XX, se inician nuevas políticas a nivel mundial que promueven la educación como punto de partida en el progreso social, económico, tecnológico y cultural de toda sociedad, dejando la consigna que esta es la clave para un futuro con bienestar. Esta nueva visión de la educación hace que los sistemas educativos de los países anglosajones en principio, europeos y posteriormente Latinos cambien la visión de la educación que se mantenía hasta entonces a ser parte de los planteamientos estratégicos en los modelos económicos, impactando en los comportamientos sociales, puesto que se expande como una necesidad que afecta todas las partes interesadas.
Así es como nace la escuela competitiva que surge a la par con los modelos de sistemas de calidad educativa en los cuales prima la calidad de los aprendizajes por encima de los conocimientos teóricos. Martínez Boom (2020) lo expresa como "esta reingeniería de la educación por el aprendizaje parece centrarse fundamentalmente en la búsqueda del máximo rendimiento posible, sociedad del rendimiento que ahora opera como una tecnología de optimización individual y social" (p. 323).

Para este siglo las tendencias de los sistemas educativos hacen parte de los cambios que se producen frecuentemente. La globalización de mercados, las tecnologías de la información, la multiculturalidad, la innovación 
en los diferentes ámbitos hacen que los sistemas educativos cambien de forma acelerada, estos cambios están encaminados en todos los sentidos, ya que se dan a través de las políticas educativas que dependen de las políticas gubernamentales o cambios que se dan de acuerdo con la común unión de territorios, o se dan al tratar de alcanzar mejores resultados en menor tiempo y con mayor evidencia. El mundo se mueve y así mismo se mueven los sistemas educativos. Mucho se ha dicho y de alguna manera se ha explicado biológicamente como los estudiantes de hoy no son como los de ahora treinta años, a tempranas edades el ser humano ha evolucionado de una manera vertiginosa la globalización y la internet, han modificado los aprendizajes en los niños. Tener la información a la mano solo con hacer un clic, hace que la educación experimente una acelerada evolución de la misma manera. Con esto nacen las nuevas tendencias en educación que se enfocan en estrategias innovadoras, flexibles, conectadas con el mundo digital, estrategias que van más de allá de seguir un plan de estudios conun currículo lleno de temáticas y estrategias que se adaptan a nuevos contextos, que no dependen deestarensu salón de clasesy que mucho menos depende de la edad de los estudiantes.

Desde este punto de vista, se hace necesaria una educación transformadora de vidas, capaz de desarrollar habilidades para un mundo continuamente cambiante, donde la competitividad es permanente. Una educación basada en los valores humanos y medio ambientales, enmarcada por la equidad, la inclusión, la calidad, pertinencia y la permanencia en el sistema, no por la obligatoriedad sino por la necesidad de ser partícipe de este nuevo mundo, enfocada en la necesidad de aprender a aprender, en el saber hacer ante las diferentes situaciones presentadas, en el autoapren- dizaje que garantice que la persona mantenga formación continua a lo largo de su vida y en el saber ser en la interacción por las diversas relaciones laborales, personales y sociales con que se deberá encontrar a lo largo de su vida.

Por todo lo anterior es como aparece en la década de los ochentas la necesidad de integrar las necesidades industriales, en cuanto mano de obra con los sistemas educativos de aquel entonces, emergen entonces como respuesta a ello las competencias.

\section{DESARROLLO}

En 2014 Espinosa et al (2014) extrae de múltiples autores la definición de aprendizajes para la vida como:

las capacidades, conocimientos, habilidades, valores, actitudes que todos los individuos necesitan para lograr su identidad, realización y desarrollo personal y proyectar un futuro mejor, para desarrollar relaciones armónicas, para participar eficazmente en los ámbitos personal, social, profesional y político y enfrentar exitosamente los desafíos de la vida diaria y a las situaciones excepcionales, para comprender el mundo e influir en él y transformarlo (p. 2042).

Al respecto se puede decir que los aprendizajes para la vida se pueden adquirir o desarrollar de diversas maneras dentro y fuera de una institución educativa y son llamados competencias. Al integrar múltiples conceptos sobre competencia esta se define como un conjunto de saberes personales con una exigencia de determinados conocimientos, habilidades, cualidades o aptitudes para ser utilizados en situaciones concretas de trabajo o educación, teniendo en cuenta recursos ambientales (rela- 
ciones, documentos, información), actuando de manera eficiente para el logro de determinada acción, en medio de comportamientos éticos y manejo inteligente de las emociones. Así mismo, las competencias se han clasificado de múltiples formas, sin embargo, sobresalen en el lenguaje común las básicas, genéricas, laborales de gestión, laborales técnicas, específica, cognitivas, procedimentales, actitudinales, intrapersonales, interpersonales, profesionales y disciplinares. Las aplicaciones de las competencias en el medio laboral o en el medio educativo determinan la mejor clasificación y sus posibles combinaciones ya que de ello depende los desempeños que se desean lograr (Contreras 2011, p.118).

Basándose en el proyecto Tunning, se han clasificado las competencias en genéricas o transversales y en específicas, es decir, propias de cada especialidad. Gracias al proyecto se determinó que los planes de estudio debían contener tanto competencias genéricas como específicas. De aquí se deriva entonces que las competencias genéricas toman diferentes nombres de acuerdo al autor, sobresalen algunos sinónimos como competencias básicas, competencias clave, competencias transversales, competencias genéricas o competencias de empleabilidad, entre otras. (Pugh y Lozano-Rodríguez 2019, p. 153).

Las competencias genéricas toman mayor relevancia hoy día, dado los nuevos contextos sociales y tecnológicos donde los nuevos profesionales y personas en edad productiva deberán enfrentar desafíos en contextos multiculturales, medioambientales, nacionales e internacionales. Las nuevas tecnologías reemplazaran la mano de obra, por ello las competencias genéricas están enfocadas al talento humano de las empresas, aunque los saberes disciplinares son importantes y las personas deben estar capacitados de manera competente en su saber hacer, los entornos multidisciplinarios cada vez más comunes en las organizaciones, requieren que las personas sean altamente efectivas a la hora de comunicarse, sepan participar en múltiples equipos, sea innovador, emprendedor, flexible, con capacidad y disposición a movilizarse, pero con fuertes valores éticos.

El proyecto Tunning determino 30 competencias genéricas, análogamente estudios en Latinoamérica han definido 27 competencias genéricas comunes a los diferentes programas universitarios y que impactan en el mundo globalizado al que deberán enfrentarse las actuales y las nuevas generaciones que se encuentran en las instituciones educativas desde los niveles básicos hasta la educación superior. Algunos estudios de estudiantes universitarios que recién ingresan a la educación superior han demostrado que las competencias genéricas deben implementarse desde grados inferiores pues estas se desarrollan a lo largo de la vida y están influenciadas por el contexto familiar, social y cultural. El desarrollo tardío de las mismas genera dificultades en los niveles superiores pues se dificultan los aprendizajes de las mismas sino se han sentado bases sólidas para ello.

Países como Nueva Zelanda, Australia, Canadá, Reino Unido entre otros datan de la necesidad de implementar las competencias genéricas en sus planes de estudio pues con esto garantizan que los estudiantes puedan tener existo en los diversos contextos laborales que les presenta el mundo cambiante de hoy. Así lo manifiestan Lozano y Herrerara (2013) (como se citó en Pugh y Lozano-Rodríguez 2019, p. 155), "el desarrollo de 
las competencias genéricas aumenta la competitividad, eficiencia y productividad de la fuerza laboral del país, proporcionando una ventaja competitiva a nivel internacional". En todos los casos las competencias genéricas están llamadas a la nueva sociedad del conocimiento donde además aparecen otros tipos de competencias dados los avances tecnológicos en los que se encuentra enmarcada la educación y son aquellas donde se emplean las nuevas tecnologías de la información.

Las treinta competencias definidas en el proyecto Tunning o veintisiete en el caso de Latinoamérica no son fáciles de implementar en el aula de clase, por lo que se ha sugerido que del listado propuesto se determinen las más importantes de acuerdo al perfil de egresado que haya determinado la institución educativa y sean desarrolladas en las asignaturas determinadas en las diferentes carreras. (Corominas, 2001; Freire, Teijeiro y Pais, 2011; Palmer, Montaño y Palou, 2009; Quintana, Raccoursier, Sánchez, Sidler y Toirkens, 2007; Torra et al., 2010, como se citó en Pugh y Lozano-Rodríguez 2019, p. 159). Esta es la estrategia que ha implementado la educación colombiana. Las competencias laborales se desarrollan de manera trasversal a las diferentes asignaturas que se encuentran reglamentadas en la ley general de educación de 1994. Con la ley general de educación de 1994, la apertura económica y las nuevas disposiciones en el mercado laboral en el país nace la necesidad de profesionales y personal calificado con habilidades no solo de conocimiento teóricos, sino que posea competencias generales y otras que hagan parte de su especialidad. Como complemento a la ola que se generaba en este tiempo sobrecalidad educativa se integran las competencias y de allí se desprenden políticas de aseguramiento de la calidad basadas en los lineamientos y estándares curriculares, como también se inicia la evaluación mediante evidencias de desempeño, todos con un enfoque basado en competencias.

En Colombia las competencias laborales se definen como aquellas que están relacionadas con la productividad y la competitividad. Conforme a ello se han emitido políticas educativas donde se determina que el sistema educativo colombiano debe desarrollar en los estudiantes las competencias básicas, ciudadanas y laborales. Las políticas de articulación de la educación con el mundo productivo están determinadas en la guía $\mathrm{N}^{\circ} 21$ del Ministerio de Educación Nacional, en ella se explicitan las competencias laborales que son clasificadas en dos grades ramas, a saber, las competencias laborales generales y las competencias laborales específicas. Las competencias laborales generales son adquiridas por los estudiantes a lo largo de los niveles educativos desde el preescolar hasta el grado noveno, mientras que las competencias laborales específicas se desarrollan en el nivel de la media (grados décimos y once).

Entre tanto, las pruebas estandarizadas aplicadas a los diferentes niveles de educación Pruebas Saber (Saber 11, Saber T y T y las Saber Pro) están enfocadas en la valoración de competencias genéricas y competencias específicas, estas últimas para los niveles técnico a superior. Las competencias genéricas se evalúan en las diferentes pruebas saber dentro de las áreas de matemáticas, lectura crítica, competencias ciudadanas, ciencias naturales e inglés, aunque según la literatura el modelo pedagógico en el que se encuentre el estudiante puede llegar a influir en el logro de los aprendizajes esperados, por lo que es importante una adecuada metodología de enseñanza basada 
en las competencias de acuerdo al plan de estudios. Queda la inquietud si las competencias básicas pueden desarrollarse mejor bajo ciertos modelos pedagógicos que otros y cuáles serían esas variables determinantes en el proceso.

Las estrategias metodológicas para el desarrollo de las competencias genéricas tienen en cuenta que los estudiantes de hoy no aprenden porque el profesor así lo determine. Ellos aprenden por un motivo, aprenden para divertirse, aprenden para sorprenderse, gustan de los retos y aprenden para enfrentarse a algo nuevo. Por todo esto, el desarrollo de las competencias genéricas en el aula requiere metodologías flexibles que sean capaces de adaptarse a cambios inesperados que puedan dar las herramientas necesarias a los ciudadanos del futuro y que le brinde las fortalezas necesarias para afrontar un mundo competitivo y lleno de contrastes emocionales, sociales y culturales, pero que además potencialice sus capacidades y tenga en cuenta sus gustos.

Como se ha visto las competencias genéricas se han implementado desde hace ya más de tres décadas, permeando todos los ámbitos de la sociedad a nivel socioeconómico, cultural social, educativo, político, laboral, tecnológico entre otros. A través de las competencias genéricas se están generando los perfiles de los egresados no solo de profesionales sino de técnicos, tecnólogos y de los mismos estudiantes de la educación básica y media. Sin embargo, existen alrededor de las mismas ciertas amenazas debido a la utilización de la palabra competencia en un lenguaje que no va más allá de su significado real, prueba de ello se encuentra en que algunos docentes desconocen que la evaluación de las competencias genéricas se encuentra incluidas dentro de las pruebas saber, creyendo aún que estas valoraciones son solo de tipo académico o cognitivo, mostrando que aún persiste la educación memorística y que está reducida a la transmisión de conocimientos. Por otro lado, ya se encuentra en fases iniciales las pruebas estandarizadas de las competencias socioemocionales muy de moda hoy día.

El objetivo de la educación con enfoque basado en competencias es generar verdaderos aprendizajes en los estudiantes al pasar de una educación tradicional basada en la transmisión de conocimientos, a aquella en la que los aprendizajes se den para la vida y para los contextos necesarios de acuerdo con las necesidades actuales de globalización y sociedad del conocimiento. Sin embargo, en medio de las políticas de calidad educativa en donde se han generado múltiples esfuerzos por aumentar la cobertura, mejorar la calidad educativa (visto desde las pruebas de desempeño), reducir la brecha social, aumentar la eficacia y pertinencia, las competencias no están siendo utilizadas para mejorar los aprendizajes en los estudiantes. Los indicadores de calidad como cobertura o deserción escolar no muestran los niveles de aprendizaje que están logrando los estudiantes. Por el contrario, aquellas pruebas como las pruebas estandarizadas o las pruebas internacionales como PISA si dan cuenta de la falta aun de comprender la necesidad de implementar las competencias en la práctica educativa. En otras palabras, la falta de aplicación de las competencias en las aulas, está generando un subdesarrollo en los aprendizajes que requieren los estudiantes y la sociedad para poder cambiar las situaciones de pobreza o de falta de oportunidades en aquellos con niveles socioeconómicos menos favorecidos. Es preocupante las afirmaciones que muestra el Banco Mundial al afirmar "que a menudo los docentes no tienen las competencias ni la 
motivación para enseñar de manera eficaz. Los docentes son el principalfactor que afecta el aprendizaje en las escuelas" (UNESCO, 2018). Si los docentes no poseen las competencias para generar aprendizajes eficaces en los estudiantes, difícilmente estos podrán adquirir las competencias necesarias para la vida.

De otro lado, aunque existen millones de personas en búsqueda de empleo, muchos empresarios afirman tener grandes dificultades para cubrir las vacantes. "Está en crisis el diálogo entre los sistemas de producción e inserción laboral y los de educación y formación profesional, lo que hace más difícil el acceso al empleo y se convierte en un freno al desarrollo del sistema productivo colombiano" así lo afirma Mejía (2016), la falta de articulación entre los diferentes niveles educativos y las necesidades del mundo productivo con los aprendizajes y competencias de los profesionales y jóvenes que se encuentran en la búsqueda de empleo. Por lo anterior, entre las estrategias educativas que se plantea el país para mejorar los niveles de aprendizaje a través de las competencias, en el Plan de Desarrollo 2018- 2022, se propone profundizar en las competencias más generales para los jóvenes de la educación media de acuerdo a sus necesidades e intereses, haciendo énfasis en aquellas que son necesarias para la Cuarta Revolución Industrial, entre las cuales están las competencias digitales, para el emprendimiento y las competencias para el desarrollo sostenible entre otras.

Mientras tanto, siguiendo la misma línea en el Plan Decenal de Educación 2018 - 2026 se plantea desarrollar en los docentes competencias en el proceso de enseñanza aprendizaje, impulsar en los estudiantes las competencias ciudadanas y profesionales, el desarrollo humano y el respeto por el medioambiente, así como también las competencias socioemocionales y todas aquellas que puedan garantizar aprendizajes para la vida en los diferentes entornos. Con estas metas y lineamientos que se han propuesto como políticas de estado se esperaría que las competencias laborales y específicas logren mejorar los desempeños en los aprendizajes de los estudiantes en todos los niveles educativos, pero que, además, mejoren las competencias que buscan las diferentes empresas que gestionan el talento humano. Las políticas también se enfocan en actualizar las competencias en docentes y directivos para garantizar que los procesos pedagógicos se desarrollen de manera armonizada por parte de todos los entes de las instituciones educativas.

\section{CONCLUSIONES}

En la actualidad las competencias genéricas que se han establecido durante la historia, están siendo sometidas a una dura prueba con la situación actual de pandemia que vive la humanidad entera. Son esas competencias para la vida las que ayudan a enfrentar todos los cambios a los que se están enfrentando todos los miembros de una comunidad educativa. desde marzo de 2020. La situación de emergencia sanitaria sacó a relucir en muchos docentes y profesionales la falta de competencias para la vida. El impacto en el aprendizaje que ha generado esta emergencia ha hecho que miles de personas se vean enfrentados a su falta de competencias sobre todo tecnológicas y digitales, sin dejar de lado que se han incrementado las situaciones psicoemocionales en adultos, niños y jóvenes, colocando en tela de juicio qué es lo que supuestamente se ha enseñado durante más de dos décadas de haberse implementado el aprendizaje basado en competencias. El Covid-19 está siendo esa herramienta de evaluación que valora el grado de solución de problemas que se tie- 
ne frente a las situaciones de la vida. Como ejemplos de esto están la creatividad que pueda implementar las personas para lograr salir de la crisis económica, el liderazgo que debe asumir un gerente, un director de una escuela para movilizar todo su equipo de trabajo y continuar en medio de la crisis, pero sobre todo está en evaluación la capacidad de saber ser, ser responsable con la vida propia y de los otros, ser disciplinados antes las normas de convivencias, ser buen ser humano.

De manera positiva la emergencia sanitaria ha generado el crecimiento de las competencias tecnológicas, la creatividad y la solución de problemas en miles de profesionales y de muchísimos maestros. La educación ha tomado un giro de 180 grados, empiezan a debatirse modelos de aprendizaje donde el estudiante este orientado a través de clases virtuales y presenciales de manera simultánea. La educación entro en una era de cambios para los cuales los docentes deberán asumir el reto de desarrollar competencias nunca antes vistas. Cabe preguntar ¿cómo movilizar todo el cuerpo docente hacia el objetivo de aprender las competencias para esta sociedad del conocimiento, en medio de la crisis que aún sigue y que ha profundizado problemas sociales, económicos y culturales en el país? ¿Cómo evitar que una vez superada la emergencia no se vuelva a la comodidad de enseñar de la misma manera como se ha hecho durante veinte años?

Es incierto el futuro, pero esperamos haber aprendido la lección, haber adquirido competencias para afrontar cambios repentinos y con ello poder evolucionar como sociedad.

\section{REFERENCIAS BIBLIOGRÁFICAS}

Banco Mundial (2018), Informe sobre el desarrollo mundial 2018: Aprender para hacer realidad la promesa de la educación, cuadernillo del "Panorama general", Banco Mundial, Washington, DC. Licencia: Creative Commons de Reconocimiento CC BY 3.0 IGO. http://repositorio.minedu.gob.pe/handle/MINEDU/5593

Contreras L, J.(2011). Formación de competencias: tendencias y desafíos en el siglo XXI. Universitas (15), p. 109-138

Espinosa Milán, L., Martínez García, B.\& Medina, F. (2014). Aprendizajes para la vida en el bachillerato, una apreciación de estudiantes del último semestre, en la ciudad de Aguascalientes. Repositorio de la Red Internacional de Investigadores en Competitividad, 8(1).

Lozano Rodríguez, A., \& Herrera Bernal, J. (2013). Diseño de programas educativos basados en competencias. Editorial Digital del Tecnológico de Monterrey.

Martínez-Boom A. (2014). ¿Para qué nos educamos hoy? Escolarización y educapital. En Noguera y Rubios (Ed). Genealogías de la pedagogía (p.307 -340). Universidad Pedagógica Nacional (2020) 
Mejía, F. J. (2016). Desafíos del Sistema Nacional de Educación Terciaria. Rutas de formación: Prácticas y Experiencias, (3), (96-97). http://dx.doi.org/10.24236/24631388.n3.2016.641

Ministerio De Educación Nacional de Colombia (2017). Plan Nacional Decenal De Educación 2016-2026: El Camino Hacia La Calidad y La Equidad.

Ministerio De Educación Nacional de Colombia (2019). Plan Nacional de Desarrollo 2018 -2022: Pacto por Colombia pacto por la equidad.

Pugh, G., \& Lozano-Rodríguez, A. (2019). El desarrollo de competencias genéricas en la educación técnica de nivel superior: un estudio de caso. Calidad en la educación, (50), 143-179). 